\title{
Evaluation of Different Phytochemicals Against BRCA2 Receptor
}

\author{
Karanpreet Singh Bhatia ${ }^{1, \dagger}$, Saksham Garg ${ }^{2, \dagger}$, Ashutosh Anand ${ }^{2, \dagger}$, Arpita Roy ${ }^{3, *(D)}$ \\ National University of Singapore, Singapore \\ Delhi Technological University, India \\ Department of Biotechnology, School of Engineering \& Technology, Sharda University, Greater Noida, India \\ the authors contributed equally; \\ Correspondence: arbt2014@gmail.com (A.R.);
}

Scopus Author ID 57203962973

Received: 15.04.2021; Revised: 20.05.2021; Accepted: 23.05.2021; Published: 9.06.2021

\begin{abstract}
Cancer is one of the most common diseases and remains a leading cause of death worldwide. Breast cancer is one of the major causes of mortality in women. It is one of the most frequent cancers among others. Due to its high mortality, it requires effective prevention. Radiotherapy, immunotherapy, and chemotherapy are the most common methods used to treat breast cancer, but these techniques adversely affect healthy cells. Therefore, an alternative therapy is required to overcome this problem. Phytochemicals from different plant sources are an alternative way for curing infectious diseases and now they are being explored for their anti-cancer therapeutics. In the present study, the BRCA2 receptor protein, which is actively involved in breast cancer expression, was chosen for the molecular docking analysis. This study focuses on evaluating phytochemical compounds from 5 different plants against BRCA2 breast cancer tumor receptors. Prior to computational analysis, a theoretical ADME study was used to rule out a few compounds. Virtual screening of these compounds identified five-hit molecules, which could be further explored in the drug discovery pipeline. Molecular docking analysis revealed that isocolumbin possesses maximum negative binding energy against BRCA2 receptors. These hits were found to be well in range in the bioavailability radar test as well.
\end{abstract}

Keywords: Phytochemicals; breast cancer; in-silico screening; docking; isocolumbinm; bioavailability.

(C) 2021 by the authors. This article is an open-access article distributed under the terms and conditions of the Creative Commons Attribution (CC BY) license (https://creativecommons.org/licenses/by/4.0/).

\section{Introduction}

Breast cancer, one of the most frequent cancers among women, affected an estimated 2.1 million women in 2018, which translates to one new case every 18 seconds [1]. Approximately $15 \%$ of women died from breast cancer [2-3]. Regardless of the income level, the burden of breast cancer is increasing in women due to the increasing and aging population [3-4]. The death rate is also varied among the cancer subtypes, with HER2 positive having the highest death rate followed by TNBC, Luminal A and Luminal B subtypes [5]. In high-income countries, cancer is often diagnosed at early stages, whereas it is diagnosed at later stages in lower- and middle-income countries. Therefore, the prognosis in high-income countries is better than that in low- and middle-income countries [4]. Some studies also reported that breast cancer is present earlier age (generally 40-50 years of age) in Asian women as compared to Western region countries' women (generally between 60-70years of age) [7-9]. 
The genetic mutation in the breast cancer susceptibility gene BRCA2 confers a predisposition to individuals' early onset of breast cancer. BRCA2 encodes BRCA2 protein, whose function is to repair DNA double-strand breaks via homologous recombination [10,11]. One of the crucial steps in homologous recombination is recruiting RAD51 recombinase to the exposed single-stranded DNA overhangs. RAD51 forms highly ordered nucleoprotein filaments that mediate the search for homologous sequences on the undamaged sister chromatids, followed by DNA strand exchange [12]. The defective cells for BRCA2 are sensitive to DNA interstrand cross-linking agents and show a decreased efficiency of DNA double-stranded break repair. They also have the inability to promote efficient restart of stalled replication forks $[13,14]$.

The development of graphics processing units and bioinformatics makes it an innovative tool to design new drugs $[15,16]$. It reduces the monetary and time costs that generally go into experiments and trials. Docking combines the chemical and physical principles with scientific calculation algorithms to generate characteristic interactions between receptor and ligand molecule [17]. Natural compounds are one of the potential sources of bioactive compounds. Plant-derived compounds possess a wide range of therapeutic activities, including anti-cancer, antibacterial, antidiabetic, etc. [18-22]. Anti-cancer activity of various plant-derived compounds has been reported in various studies [23-26]. Therefore, this study focuses on evaluating phytochemical compounds from 5 different plants, namely - Tinospora cordifolia, Ocimum tenuiflorum, Podophyllum hexandrum Andrographis paniculate, and Beta vulgaris, against BRCA2 breast cancer tumor receptor.

\section{Materials and Methods}

\subsection{Protein/Macromolecule.}

PALB2/BRCA2 complex was used in this study. The 3-D structure (PDB id: 3EU7) of the complex was retrieved from RCSB PDB in PDB file format (https://www.rcsb.org/). PALB2/BRCA2 complex has 6 amino acid residues in the active site, namely: TYR1064, PHE1071, LEU1092, TYR1108, LEU1142, and LEU1143. The active site of the complex was involved in binding with GOL (Glycerol) inhibitor, as seen in Figure 1 [27]

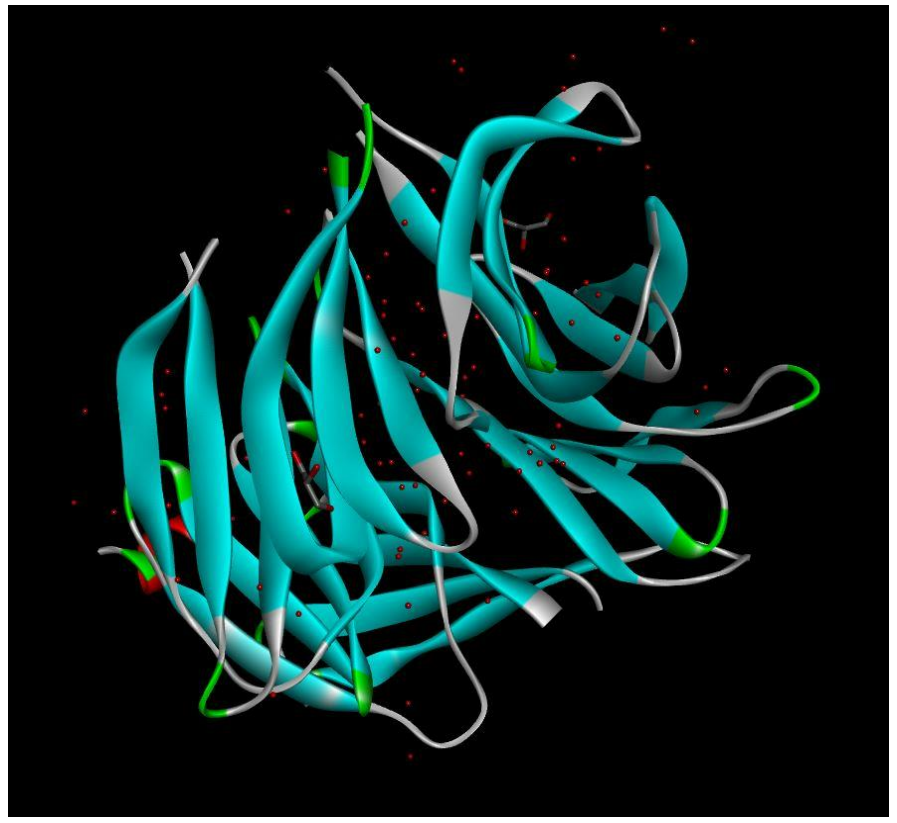

Figure 1. 3-D structure of PALB2/BRCA2 complex with GOL (Glycerol) inhibitor. 


\subsection{Ligands.}

A total of 63 bioactive compounds were used as ligands. These selected compounds are from 5 different plants, namely Tinospora cordifolia, Ocimum tenuiflorum, Podophyllum hexandrum, Andrographis paniculate, and Beta vulgaris. Ligand structures were taken from PubChem databank in .sdf format. However, for docking purposes, these structures were converted to .pdb format using Biovia Discovery Studio Visualizer v20.1.0.19295 (https://pubchem.ncbi.nlm.nih.gov/).

\subsection{ADME analysis.}

A web tool named SwissADME was used for the initial screening of the shortlisted compound based on Lipinski's rule of five. To qualify as a potential ligand, this role states that a compound should have molar weight $<500 \mathrm{Da}$; molar refractivity between 40 to $130 \mathrm{~m} 3 \mathrm{~mol}$ 1; high lipophilicity, i.e., $\log \mathrm{P}<5$; hydrogen bond donor less than 5 and acceptors less than 10 . Any compound violating two or more of the parameters mentioned above was ruled out from further studies.

\subsection{Molecular docking.}

Autodock 4.2 was used to get the docked protein-ligand complex. The structure of every ligand and protein underwent optimization before docking. From the 3-D structure of the protein, water molecules, as well as inhibitors, were removed. Further, the addition of polar hydrogen bonds, Kollman charges and Gasteiger charges concluded the step of protein and ligand preparation. A grid box of dimension $54 \times 40 \times 40$ with the spacing of $0.375 \AA$ was made around the protein's binding site. The grid was centered at $6.751,-7.741$ and 7.743 in $\mathrm{x}, \mathrm{y}$ and $\mathrm{z}$, respectively. The genetic algorithm was used as the search parameter and Lamarckian GA was used to handle the output. Docking Log File (DLG) was used for further analysis. The resulting DLG file reports a total of 10 conformations for each ligand. However, the most stable conformation, i.e., the conformation with the most negative binding energy, was selected as the final docking complex. This complex was converted to a 2-D structure to study the protein and ligand interactions at the binding site.

\subsection{Bioavailability radar.}

The Drug-likeliness of the compounds having binding energy less than the control was further analyzed. Six physiochemical properties: size, polarity, lipophilicity, solubility, flexibility, and saturation were considered to prepare a bioavailability radar via the SwissADME web tool. The shaded region shown in the radar chart represents the optimal values for the aforementioned physicochemical properties. Compounds exhibiting large deviations from these values suggest that the compound is not orally bioactive.

\subsection{PASS web server.}

PASS stands for Prediction of Activity Spectra for Substances. It is a tool used to evaluate the biological potential of an organic drug-like molecule. PASS reports predictions of various biological activities based on the structure of the compound. It calculates the probability $\mathrm{P}_{\mathrm{a}}$ and $\mathrm{P}_{\mathrm{i}}$, which describes whether the given compound belongs to the active subclass or inactive subclass, respectively. 


\section{Results and Discussion}

\subsection{ADME analysis.}

In order to estimate the drug-likeliness, Lipinski's rule was used on all the selected 63 bioactive compounds. Lipinski's rule is used to evaluate a bioactive compound's druglikeliness and assist in ruling out few compounds based on their chemical and physical properties. However, Lipinski's rule only reports whether a compound is likely to be orally active in humans. Therefore, a compound qualifying the parameters doesn't make it a drug molecule. Compounds that violate two or more than two parameters were not considered for further studies. 18 bioactive compounds were withheld from further studies. Table 1 enlists the compounds considered for further studies.

Table 1. ADME analysis of bioactive compounds.

\begin{tabular}{|c|c|c|c|c|c|c|c|}
\hline Compound Name & PubChem ID & $\begin{array}{c}\text { Lipophilicity } \\
(\log P<5)\end{array}$ & $\begin{array}{c}\text { H Bond } \\
\text { Donor }(<5)\end{array}$ & $\begin{array}{c}\text { H Bond } \\
\text { Acceptor }(<10)\end{array}$ & $\begin{array}{c}\text { Molar } \\
\text { Refractivity } \\
(\mathbf{4 0 - 1 3 0 )} \\
\end{array}$ & $\begin{array}{c}\text { Molecular } \\
\text { Weight } \\
(<500 \text { Da })\end{array}$ & Violation(s) \\
\hline Berberin & 2353 & 3.6 & 0 & 4 & 94.87 & 336.4 & 0 \\
\hline Choline & 305 & 3.6 & 1 & 1 & 29.69 & 104.17 & 1 \\
\hline Magnoflorine & 73337 & 2.7 & 2 & 4 & 101.87 & 342.4 & 0 \\
\hline Tinosporin & 122206355 & 1.1 & 2 & 8 & 97.66 & 406.4 & 0 \\
\hline Palmatine & 19009 & 3.7 & 0 & 4 & 101.8 & 352.4 & 0 \\
\hline Isocolumbin & 24721165 & 2.2 & 1 & 6 & 90.1 & 358.4 & 0 \\
\hline Aporphine & 114911 & 3 & 0 & 1 & 78.97 & 235.32 & 0 \\
\hline Jatrorrhizine & 72323 & 3.4 & 1 & 4 & 97.33 & 338.4 & 0 \\
\hline Tetrahydropalmatine & 5417 & 3.2 & 0 & 5 & 103.99 & 355.4 & 0 \\
\hline Palmarin & 442068 & 1.3 & 1 & 7 & 89.54 & 374.4 & 0 \\
\hline Tembetarine & 167718 & 3 & 2 & 4 & 102.87 & 344.4 & 0 \\
\hline Eugenol & 3314 & 2 & 1 & 2 & 49.06 & 164.2 & 0 \\
\hline Methyl Eugenol & 7127 & 2.5 & 0 & 2 & 53.53 & 178.23 & 0 \\
\hline Cirsilineol & 162464 & 2.9 & 2 & 7 & 91.44 & 344.3 & 0 \\
\hline Cirsimartin & 1888323 & 2 & 2 & 6 & 84.95 & 314.29 & 0 \\
\hline Isothymusin & 630253 & 2.6 & 3 & 7 & 86.97 & 330.29 & 0 \\
\hline Apigenin & 5280443 & 1.7 & 3 & 5 & 73.99 & 270.24 & 0 \\
\hline Rosemeric Acid & 5281792 & 2.36 & 5 & 8 & 91.4 & 360.31 & 0 \\
\hline Luteolin & 5280445 & 2.53 & 4 & 6 & 76.01 & 286.24 & 0 \\
\hline Bornyl Acetate & 6448 & 4.3 & 0 & 2 & 56.33 & 196.29 & 0 \\
\hline Myrtenal & 61130 & 2.98 & 0 & 1 & 45.42 & 150.22 & 0 \\
\hline Neral & 643779 & 3.03 & 0 & 1 & 49.44 & 152.23 & 0 \\
\hline Alpha Elemene & 80048 & 5 & 0 & 0 & 70.42 & 204.35 & 0 \\
\hline Podophyllotixin & 10607 & 2.01 & 1 & 8 & 103.85 & 414.41 & 0 \\
\hline $\begin{array}{l}\text { 4- } \\
\text { dimethylpodophylloto } \\
\text { xin }\end{array}$ & 122667 & 1.68 & 2 & 8 & 99.38 & 400.38 & 0 \\
\hline Quercetin & 5280343 & 1.54 & 5 & 7 & 78.03 & 302.24 & 0 \\
\hline Kaempferol & 5280863 & 1.9 & 4 & 6 & 76.01 & 286.24 & 0 \\
\hline Andrographolide & 5318517 & 2.16 & 3 & 5 & 95.21 & 350.45 & 0 \\
\hline $\begin{array}{l}\text { Isopropylideneandrog } \\
\text { rapholide }\end{array}$ & 71589898 & 3.17 & 1 & 5 & 107.41 & 390.51 & 0 \\
\hline $\begin{array}{l}\text { 14-deoxy-11,12- } \\
\text { didehydroandrograph } \\
\text { olide }\end{array}$ & 5708351 & 3.23 & 2 & 4 & 93.58 & 332.43 & 0 \\
\hline $\begin{array}{l}\text { Dehydroandrographol } \\
\text { ide }\end{array}$ & 6473762 & 3.38 & 2 & 4 & 93.58 & 332.43 & 0 \\
\hline Moslosooflavone & 188316 & 3.32 & 1 & 5 & 82.93 & 298.29 & 0 \\
\hline $\begin{array}{l}\text { 19-O-acetyl-14- } \\
\text { deoxy-11,12- }\end{array}$ & 46179874 & 3.26 & 1 & 5 & 103.32 & 374.47 & 0 \\
\hline
\end{tabular}




\begin{tabular}{|c|c|c|c|c|c|c|c|}
\hline Compound Name & PubChem ID & $\begin{array}{c}\text { Lipophilicity } \\
(\log P<5)\end{array}$ & $\begin{array}{c}\text { H Bond } \\
\text { Donor }(<5)\end{array}$ & \begin{tabular}{c|} 
H Bond \\
Acceptor $(<\mathbf{1 0})$
\end{tabular} & $\begin{array}{c}\text { Molar } \\
\text { Refractivity } \\
(40-130)\end{array}$ & $\begin{array}{l}\text { Molecular } \\
\text { Weight } \\
\text { (<500 Da) }\end{array}$ & Violation(s) \\
\hline \multicolumn{8}{|l|}{$\begin{array}{l}\text { didehydroandrograph } \\
\text { olide }\end{array}$} \\
\hline $\begin{array}{l}\text { (8S,12R)- } \\
\text { Isoandrographolide }\end{array}$ & 101569019 & 2.34 & 2 & 5 & 93.55 & 350.45 & 0 \\
\hline $\begin{array}{l}14- \\
\text { acetylandrographolide }\end{array}$ & 71589914 & 2.74 & 2 & 6 & 104.95 & 392.49 & 0 \\
\hline Azatoxin & 125383 & 2.94 & 2 & 5 & 106.4 & 380.39 & 0 \\
\hline Betaine & 247 & 0 & 0 & 2 & 28.35 & 117.15 & 1 \\
\hline Ferulic Acid & 445858 & 1.51 & 2 & 4 & 51.63 & 194.18 & 0 \\
\hline Caffeic Acid & 689043 & 1.15 & 3 & 4 & 47.16 & 180.16 & 0 \\
\hline p-Coumaric Acid & 637542 & 1.46 & 2 & 3 & 45.13 & 164.16 & 0 \\
\hline Syringic Acid & 10742 & 1.04 & 2 & 5 & 48.41 & 198.17 & 0 \\
\hline Rhamnetin & 5281691 & 1.87 & 4 & 7 & 82.5 & 316.26 & 0 \\
\hline Rhamnocitirin & 5320946 & 2.22 & 3 & 6 & 80.48 & 300.26 & 0 \\
\hline Betaxanthin & 135926572 & 1.38 & 2 & 7 & 94.99 & 358.35 & 0 \\
\hline Orientin & 5281675 & 0 & 8 & 11 & 108.63 & 448.38 & 2 \\
\hline Vicenin-2 & 3084407 & -2.26 & 11 & 15 & 139.23 & 594.52 & 4 \\
\hline Ursolic Acid & 64945 & 7.34 & 2 & 3 & 136.91 & 456.7 & 2 \\
\hline $\begin{array}{l}\text { Podophyllotoxin } \\
\text { glucoside }\end{array}$ & 161177 & 0.42 & 4 & 13 & 136.23 & 576.55 & 2 \\
\hline Etoposide & 36462 & 0.6 & 3 & 13 & 139.11 & 588.56 & 3 \\
\hline Teniposide & 452548 & 1.24 & 3 & 13 & 156.66 & 656.65 & 3 \\
\hline NK611 & 9852558 & 2.12 & 2 & 13 & 157.42 & 652.09 & 3 \\
\hline GL331 & 148091 & 3.88 & 2 & 9 & 135.86 & 520.49 & 2 \\
\hline TOP-53 & 177859 & 3.02 & 1 & 9 & 137.66 & 512.59 & 2 \\
\hline Etoposide Phosphate & 6918092 & 0 & 4 & 16 & 149.76 & 668.54 & 3 \\
\hline Tafluposide & 9877073 & 4.84 & 2 & 30 & 220.84 & 1116.71 & 3 \\
\hline Beta-Sitosterol & 222284 & 9.34 & 1 & 1 & 133.23 & 414.71 & 2 \\
\hline Stigmasterol & 5280794 & 8.56 & 1 & 1 & 132.75 & 412.69 & 2 \\
\hline Rutin & 5280805 & 0 & 10 & 16 & 141.38 & 610.52 & 4 \\
\hline Astragalin & 5282102 & 0.72 & 7 & 11 & 108.13 & 448.38 & 2 \\
\hline Oleanolic Acid & 10494 & 7.49 & 2 & 3 & 136.65 & 456.7 & 2 \\
\hline Beta-Carotene & 5280489 & 13.54 & 0 & 0 & 184.43 & 536.87 & 3 \\
\hline Lutein & 5281243 & 11.01 & 2 & 2 & 186.76 & 568.87 & 3 \\
\hline
\end{tabular}

\section{(A)14acetyl}

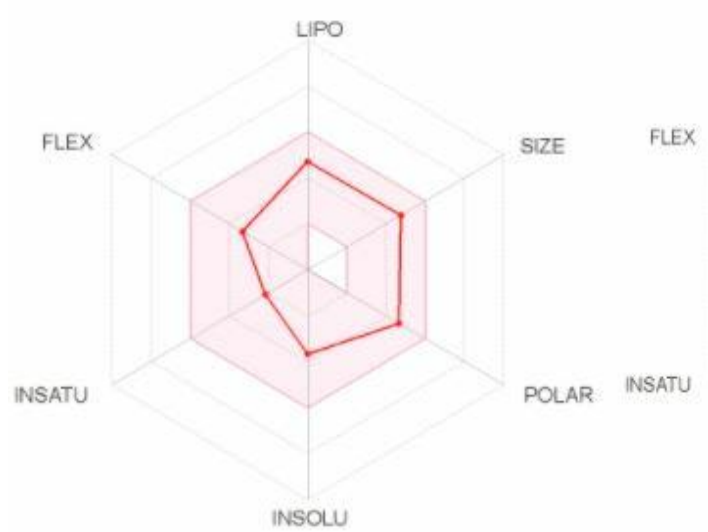

(B)14deoxy

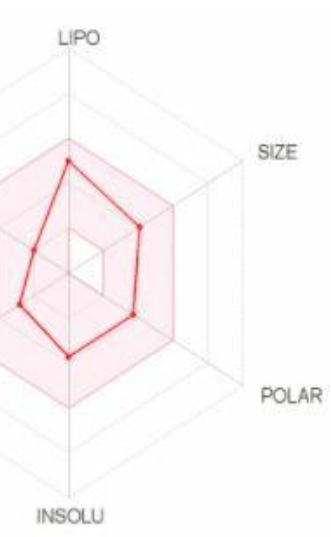




\section{(C)190Acetyl}

(D)Isocolumbin

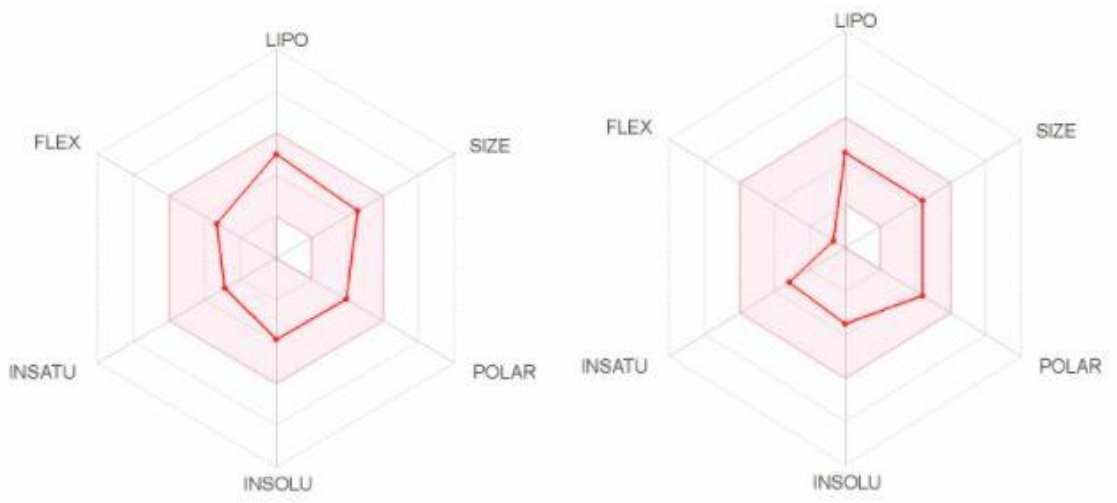

(E)Isoprpoyl

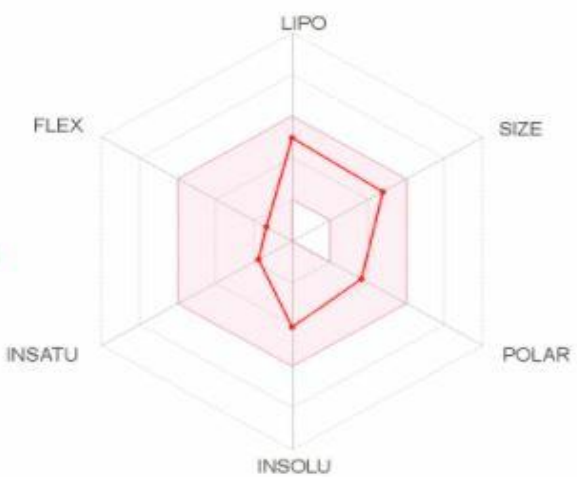

Figure 2. Bioavailability radar representation of five selected hits. The colored zone represents the preferable physicochemical space for oral bioavailability. LIPO stands for Lipophilicity: $-0.7<$ XLOGP3<+5.0. SIZE: 150 $\mathrm{g} / \mathrm{mol}<\mathrm{TPSA}<130 \AA 2$ 2. INSOLU stands for Insolubility: $0<\operatorname{LogS}(\mathrm{ESOL})<$ fraction Csp3 < Number of rotatable bonds $<9$.

Table 2. Virtual screening results of the drug library. The top five molecules were further analyzed for their

\begin{tabular}{c|c|c|c|c|c} 
& \multicolumn{7}{c}{$\begin{array}{c}\text { bioavailability using the pass webserver. } \\
\text { Compounds }\end{array}$} & $\begin{array}{c}\text { Binding } \\
\text { Energy }\end{array}$ & $\begin{array}{c}\text { Ligand } \\
\text { Efficiency }\end{array}$ & $\begin{array}{c}\text { Inhibition } \\
\text { constants (uM) }\end{array}$ & $\begin{array}{c}\text { Intermolecular } \\
\text { energy }\end{array}$ & $\begin{array}{c}\text { vdw H bond desolvation } \\
\text { energy }\end{array}$ \\
\hline Isocolumbin & -8.73 & -0.34 & 0.39684 & -9.33 & -9.37 \\
\hline $\begin{array}{c}19-O-\text {-acetyl-14- } \\
\text { deoxy-11,12- } \\
\text { didehydroandrog } \\
\text { rapholide }\end{array}$ & -8.5 & -0.31 & 0.5926 & -10.29 & -10.18 \\
\hline $\begin{array}{c}\text { Isopropylidenean } \\
\text { drographolide }\end{array}$ & -7.99 & -0.29 & 1.39 & -8.88 & -8.79 \\
\hline $\begin{array}{c}14- \\
\text { acetylandrograph } \\
\text { olide }\end{array}$ & -7.61 & -0.27 & 2.64 & -9.7 & -9.58 \\
\hline $\begin{array}{c}14-\text { deoxy-11,12- } \\
\text { didehydroandrog } \\
\text { rapholide }\end{array}$ & -7.49 & -0.31 & 3.25 & -8.98 & -8.92 \\
\hline $\begin{array}{c}\text { Dehydroandrogra } \\
\text { pholide }\end{array}$ & -7.35 & -0.31 & 4.07 & -8.84 & -8.73 \\
\hline Berberin & -7.32 & -0.29 & 4.3 & -7.92 & -7.86 \\
\hline Aporphine & -7.32 & -0.41 & 4.32 & -7.32 & -6.84 \\
\hline Andrographolide & -7.32 & -0.29 & 4.34 & -9.11 & -8.94
\end{tabular}


https://doi.org/10.33263/BRIAC122.16701681

\begin{tabular}{|c|c|c|c|c|c|}
\hline Compounds & $\begin{array}{l}\text { Binding } \\
\text { Energy }\end{array}$ & $\begin{array}{l}\text { Ligand } \\
\text { Efficiency }\end{array}$ & $\begin{array}{c}\text { Inhibition } \\
\text { constants (uM) }\end{array}$ & $\begin{array}{c}\text { Intermolecular } \\
\text { energy }\end{array}$ & $\begin{array}{c}\text { vdw } \mathrm{H} \text { bond desolvation } \\
\text { energy }\end{array}$ \\
\hline $\begin{array}{c}\text { (8S,12R)- } \\
\text { Isoandrographoli } \\
\text { de }\end{array}$ & -7.28 & -0.29 & 4.64 & -8.47 & -8.37 \\
\hline Azatoxin & -7.28 & -0.26 & 4.6 & -8.47 & -8.43 \\
\hline Palmatine & -7.02 & -0.27 & 7.21 & -8.21 & -8.04 \\
\hline $\begin{array}{c}4- \\
\text { demethylpodoph } \\
\text { yllotoxin (CID: } \\
122667)\end{array}$ & -6.85 & -0.24 & 9.46 & -8.35 & -8.31 \\
\hline $\begin{array}{c}\text { Tetrahydropalma } \\
\text { tine }\end{array}$ & -6.83 & -0.26 & 9.84 & -8.02 & -7.63 \\
\hline Magnoflorine & -6.68 & -0.27 & 12.8 & -7.87 & -7.42 \\
\hline Tinosporin & -6.66 & -0.23 & 13.2 & -8.15 & -7.93 \\
\hline Palmarin & -6.54 & -0.24 & 16.13 & -7.13 & -7.17 \\
\hline $\begin{array}{l}\text { Podophyllotoxin } \\
\text { (CID: 10607) }\end{array}$ & -6.53 & -0.22 & 16.23 & -8.03 & -7.98 \\
\hline $\begin{array}{l}\text { Apigenin (CID: } \\
5280443)\end{array}$ & -6.51 & -0.33 & 17.03 & -7.7 & -7.53 \\
\hline Cirsilineol & -6.46 & -0.26 & 18.37 & -8.25 & -7.89 \\
\hline $\begin{array}{c}\text { Alpha Elemene } \\
\text { (CID: 80048) }\end{array}$ & -6.42 & -0.43 & 19.84 & -7.01 & -7 \\
\hline Cirsimaritin & -6.38 & -0.28 & 21.03 & -7.87 & -7.8 \\
\hline Jatrorrhizine & -6.25 & -0.25 & 26.06 & -7.45 & -7.39 \\
\hline Tembetarine & -6.24 & -0.25 & 26.65 & -8.03 & -7.45 \\
\hline Moslosooflavone & -6.24 & -0.28 & 26.75 & -7.43 & -7.42 \\
\hline $\begin{array}{c}\text { Kaempferol } \\
\text { (CID: 5280863) }\end{array}$ & -6.23 & -0.3 & 27.28 & -7.72 & -7.57 \\
\hline Rhamnocitrin & -5.96 & -0.27 & 42.57 & -7.45 & -7.38 \\
\hline $\begin{array}{c}\text { Luteolin (CID: } \\
5280445) \\
\end{array}$ & -5.95 & -0.28 & 43.63 & -7.44 & -7.18 \\
\hline $\begin{array}{c}\text { Quercetin (CID: } \\
5280343)\end{array}$ & -5.91 & -0.27 & 46.71 & -7.7 & -7.43 \\
\hline Isothymusin & -5.74 & -0.24 & 61.7 & -7.53 & -7.45 \\
\hline $\begin{array}{l}\text { Bornyl acetate } \\
\text { (CID: 6448) }\end{array}$ & -5.67 & -0.41 & 69.99 & -6.26 & -6.25 \\
\hline Rhamnetin & -5.48 & -0.24 & 96.05 & -7.27 & -7.07 \\
\hline Betaxanthin & -5.41 & -0.21 & 108.89 & -7.79 & -8.43 \\
\hline $\begin{array}{l}\text { Rosmarinic Acid } \\
\text { (CID: 5281792) }\end{array}$ & -5.35 & -0.21 & 119.52 & -8.93 & -8.87 \\
\hline $\begin{array}{c}\text { Myrtenal (CID: } \\
61130) \\
\end{array}$ & -5.34 & -0.49 & 121.98 & -5.64 & -5.64 \\
\hline Methyl Eugenol & -4.42 & -0.34 & 572.61 & -5.62 & -5.6 \\
\hline $\begin{array}{l}\text { Neral (CID: } \\
643779)\end{array}$ & -4.34 & -0.39 & 659.03 & -5.53 & -5.52 \\
\hline Eugenol & -4.27 & -0.36 & 744.04 & -5.46 & -5.41 \\
\hline Caffeic Acid & -3.95 & -0.3 & 1280 & -5.44 & -5.45 \\
\hline p-coumaric acid & -3.89 & -0.32 & 1410 & -5.08 & -5.29 \\
\hline ferulic acid & -3.72 & -0.27 & 1860 & -5.22 & -5.77 \\
\hline Syringic acid & -3.33 & -0.24 & 3600 & -4.83 & -5.16 \\
\hline Choline & -2.78 & -0.4 & 9210 & -3.67 & -3.3 \\
\hline Betaine & -2.56 & -0.32 & 13230 & -3.16 & -2.59 \\
\hline
\end{tabular}




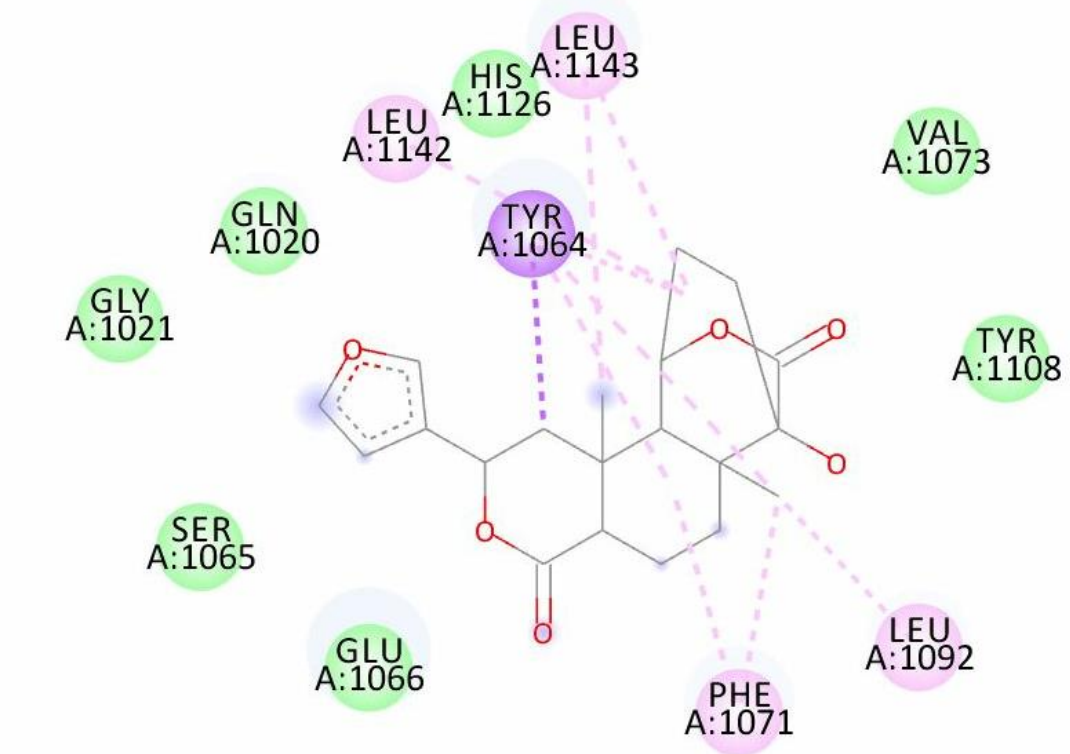

Interactions

$\square$ van der Waals
Pi-Sigma

Figure 3. Interaction of Isocolumbin with residues of 3EU7.

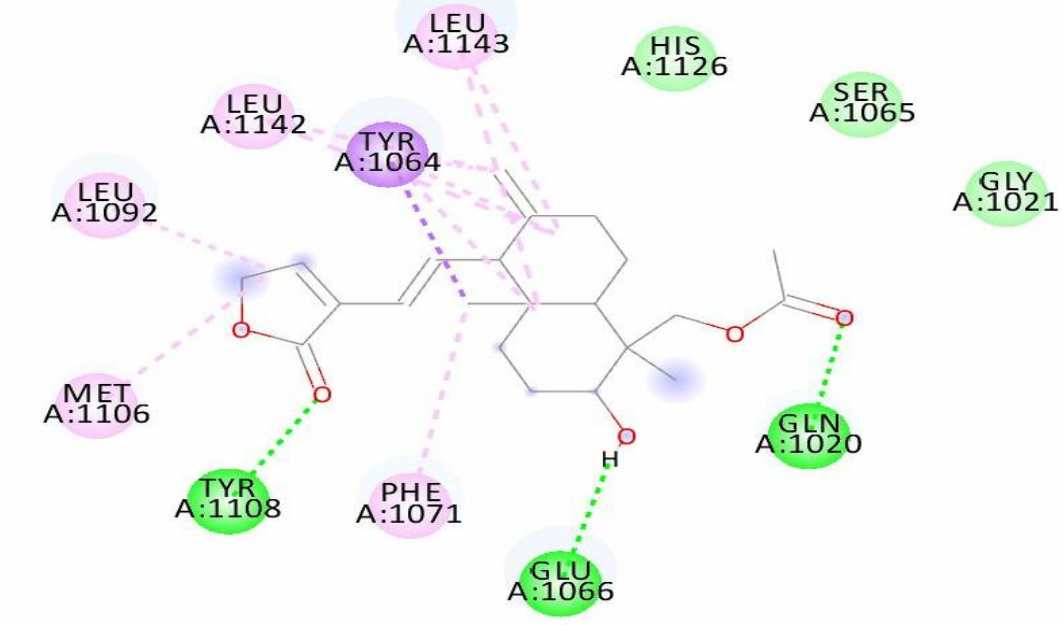

Interactions

van der Waals

Conventional Hydrogen Bond

Pi-Sigma

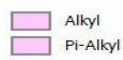

Figure 4. Interaction of 19-O-acetyl-14-deoxy-11,12-didehydroandrographolide with residues of 3EU7. 


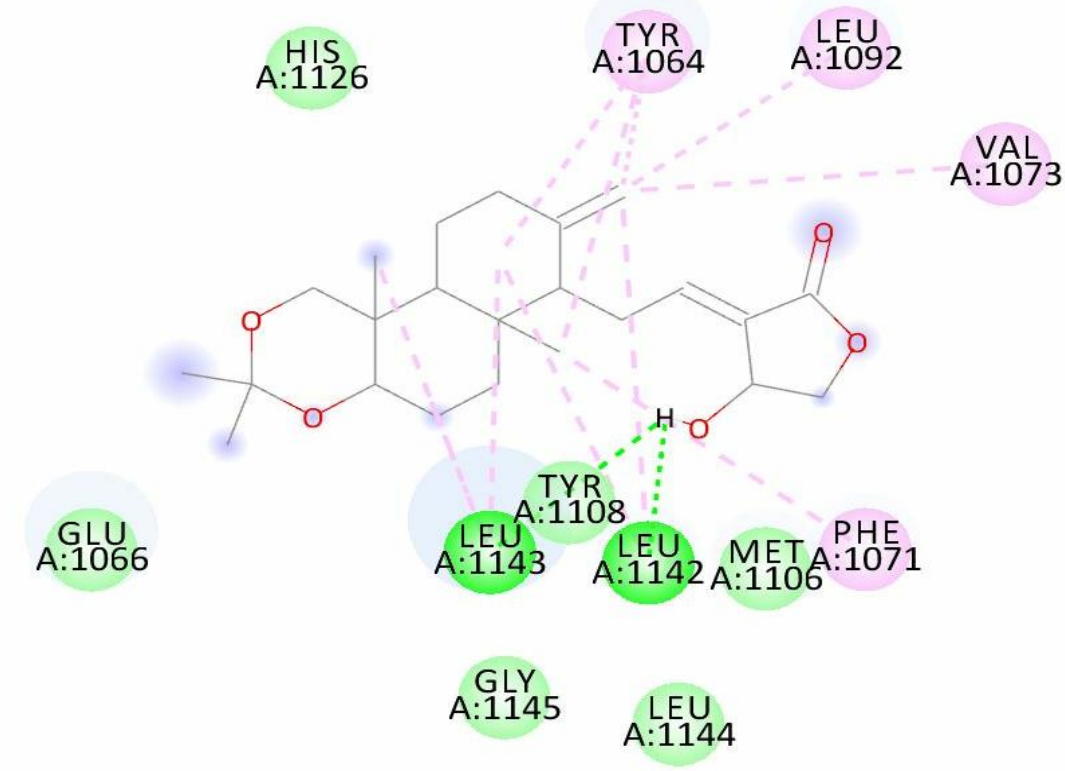

Interactions

$\square$ van der wats

Conventional Hydrogen Bond

Alkyl

Figure 5. Interaction of Isopropylideneandrographolide with residues of 3EU7.

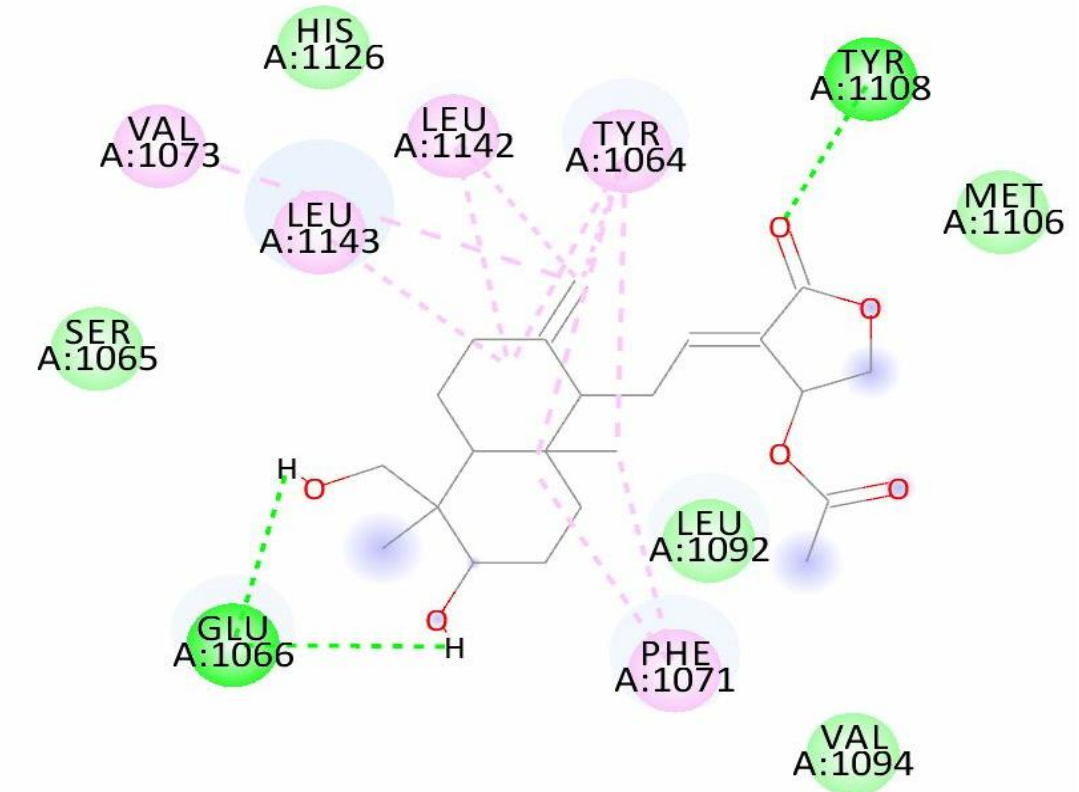

Interactions

van der Waals

Conventional Hydrogen Bond

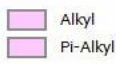

Figure 6. Interaction of 14-acetylandrographolide with residues of 3EU7. 


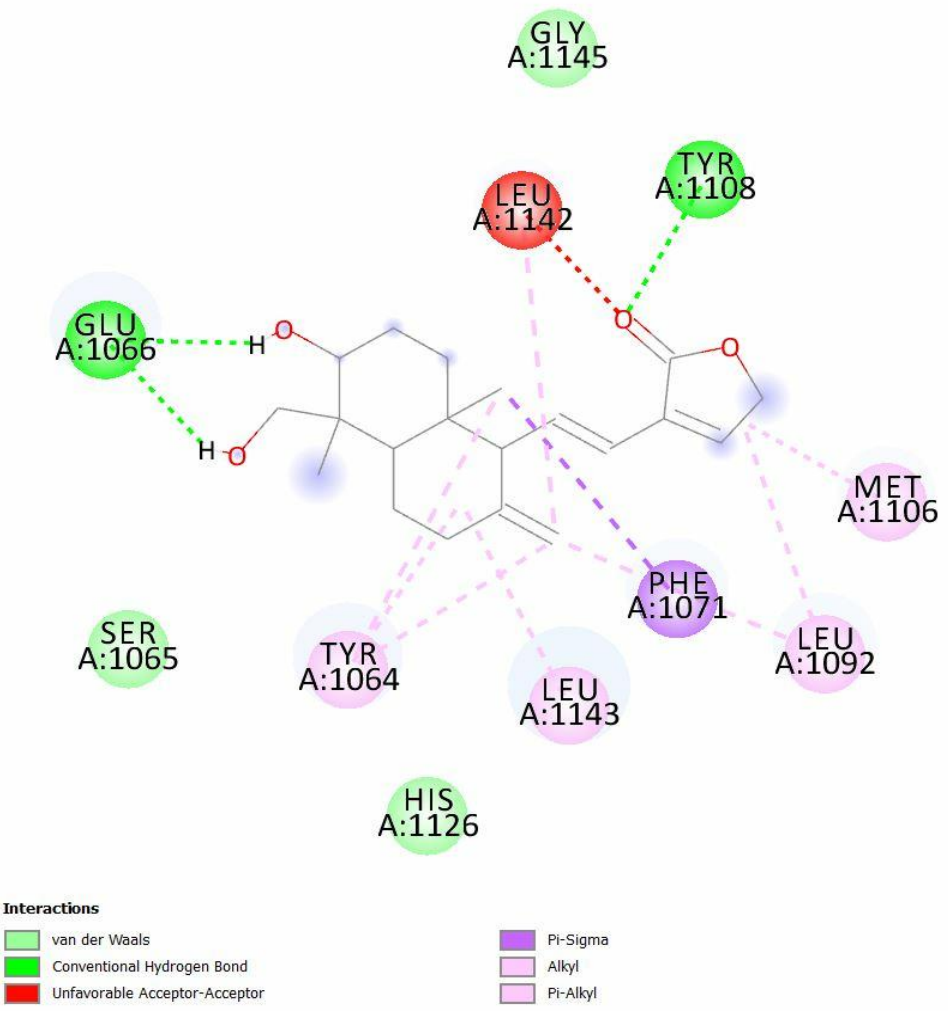

Figure 7. Interaction of 14-deoxy-11,12-didehydroandrographolide with residues of 3EU7.

Table 3. Results of Bioavailability prediction of the selected compounds.

\begin{tabular}{c|c|c|c} 
Compound & Biological activity & Pa & Pi \\
\hline $\begin{array}{c}\text { Isocolumbin } \\
\begin{array}{c}\text { 11,12- } \\
\text { didehydroandrographolide }\end{array}\end{array}$ & Antineoplastic & 0.882 & 0.005 \\
\hline $\begin{array}{c}\text { Isopropylideneandrographol } \\
\text { ide }\end{array}$ & Antineoplastic & 0.904 & 0.005 \\
\hline $\begin{array}{c}\text { 14-acetylandrographolide } \\
\text { 14-deoxy-11,12- } \\
\text { didehydroandrographolide }\end{array}$ & Antineoplastic & 0.954 & 0.004 \\
\hline
\end{tabular}

3.2. Pa- the probability that the compound is active and $P i$ - the probability that the compound is inactive.

The compounds which passed the Lipinski test were virtually screened using AutoDock and results are reported in Table 2. The top five hits with binding energy less than or equal to $-7.49 \mathrm{kcal} / \mathrm{mol}$ were selected for bioavailability prediction, which results have been shown in Table 3. Drug likeness of these five hits was also evaluated using SWISSADME and the results are given in Figure 2. Figure 2 demonstrates that these five hits possess high drug-likeness as all of these compounds show ADME properties under the range of standard oral drugs. Results in Table 3 predict the biological activity of these hits. For a molecule to be biologically active, its $\mathrm{Pa}$ should be greater than Pi and all the five hits satisfied this condition. As reported by Baseer et al., [28], the amino acid residues which show interaction in BRCA2 are TYR1064, PHE1071, LEU1092, TYR1108, LEU1142, and LEU1143, with PHE1071 being the most important one. The top five hits identified in this study also show interaction with these residues, as depicted in Figure 3-7, emphasizing the relevance of these natural compounds. In all of these compounds, pi-alkyl interaction was majorly found in between the ligand molecule and PHE1071. Interaction with other residues is also quite similar in these hits, demonstrating 
the importance of these interactions as all of these molecules have relatively better binding energy and constant inhibitory values.

\section{Conclusions}

Many researchers have explored BRCA2 and various drugs have been reported for the treatment of breast cancer manifestations with BRCA2 complexities. Side effects of the reported drugs, a resurgence of the tumor and resistance to the chemotherapy regime are some reasons for the ongoing research for the development of new compounds to tackle these issues. Natural products could answer these problems; therefore, in this study, the top five hits which we have identified after the Lipinski test, virtual screening and bioavailability prediction can further be analyzed in MD simulations and could serve as promising lead molecules which could be optimized and finally tested in clinical settings for their efficacy and safety.

\section{Funding}

Dr. Arpita Roy is thankful to Sharda University for providing seed fund (Seed fund-4 2001 (SUSF2001/12)).

\section{Acknowledgments}

We acknowledge our university for providing all the necessary facilities.

\section{Conflicts of Interest}

The authors declare no conflict of interest.

\section{References}

1. Duche, H.; Tsegay, A.T.; Tamirat, K.S. Identifying Risk Factors of Breast Cancer Among Women Attending Selected Hospitals of Addis Ababa City: Hospital-Based Unmatched Case-Control Study. Breast Cancer: Targets and Therapy 2021, 13, 189-197, https://doi.org/10.2147/BCTT.S293867.

2. Moga, M.A.; Dimienescu, O.G.; Bălan, A.; Dima, L.; Toma, S.I.; Bîgiu, N.F.; Blidaru, A. Pharmacological and therapeutic properties of Punica granatum phytochemicals: Possible roles in breast cancer. Molecules 2021, 26, 1054-1074, https://doi.org/10.3390/molecules26041054.

3. Selvakumar, P.; Badgeley, A.; Murphy, P.; Anwar, H.; Sharma, U.; Lawrence, K.; Lakshmikuttyamma, A. Flavonoids and other polyphenols act as epigenetic modifiers in breast cancer. Nutrients 2020, 12, 761-778, https://doi.org/10.3390/nu12030761.

4. Harbeck, N.; Penault-Llorca, F.; Cortes, J.; Gnant, M.; Houssami, N.; Poortmans, P.; Ruddy, K.; Tsang, J.; Cardoso, F. Breast cancer. Nature Reviews Disease Primers 2019, 5, 1-31, https://doi.org/10.1038/s41572019-0111-2.

5. Ren, J.X.; Gong, Y.; Ling, H.; Hu, X.; Shao, Z.M. Racial/ethnic differences in the outcomes of patients with metastatic breast cancer: contributions of demographic, socioeconomic, tumor and metastatic characteristics. Breast Cancer Research and Treatment 2019, 173, 225-237, https://doi.org/10.1007/s10549-018-4956-y.

6. Allemani, C.; Weir, H.K.; Carreira, H.; Harewood, R.; Spika, D.; Wang, X.S.; Bannon, F.; Ahn, J.V.; Johnson, C.J.; Bonaventure, A.; Marcos-Gragera, R.; Stiller, C.; Azevedo E Silva, G.; Chen, W.Q.; Ogunbiyi, O.J.; Rachet, B.; Soeberg, M.J.; You, H.; Matsuda, T.; Miles, S.A. Global surveillance of cancer survival 19952009: Analysis of individual data for 25676887 patients from 279 population-based registries in 67 countries (CONCORD-2). The Lancet 2015, 385, 977-1010, https://doi.org/10.1016/S0140-6736(14)62038-9.

7. Agarwal, G.; Pradeep, P.V.; Aggarwal, V.; Yip, C.H.; Cheung, P.S.Y. Spectrum of breast cancer in Asian women. World Journal of Surgery 2007 31, 1031-1040, https://doi.org/10.1007/s00268-005-0585-9.

8. Leong, S.P.L.; Shen, Z.Z.; Liu, T.J.; Agarwal, G.; Tajima, T.; Paik, N.S.; Sandelin, K.; Derossis, A.; Cody, H.; Foulkes, W.D. Is Breast cancer the same disease in Asian and Western countries? World Journal of 
Surgery 2010, 34, 2308-2324, https://doi.org/10.1007/s00268-010-0683-1.

9. Raina, V.; Bhutani, M.; Bedi, R.; Sharma, A.; Deo, S.V.S.; Shukla, N.K.; Mohanti, B.K.; Rath, G.K. Clinical features and prognostic factors of early breast cancer at a major cancer center in North India. Indian Journal of Cancer 2005, 42, 36-41, https://doi.org/10.4103/0019-509x.15099.

10. Roy, R.; Chun, J.; Powell, S.N. BRCA1 and BRCA2: Different roles in a common pathway of genome protection. In Nature Reviews Cancer, 2012, 12, 68-78, https://doi.org/10.1038/nrc3181.

11. Prabhavathi, H.; Dasegowda, K.R.; Renukananda, K.H.; Lingaraju, K.; Naika, H.R. Exploration and evaluation of bioactive phytocompounds against BRCA proteins by in silico approach. Journal of Biomolecular Structure and Dynamics 2020, 1-15, https://doi.org/10.1080/07391102.2020.1790424.

12. Shahid, T.; Soroka, J.; Kong, E.H.; Malivert, L.; McIlwraith, M.J.; Pape, T.; West, S.C.; Zhang, X. Structure and mechanism of action of the BRCA2 breast cancer tumor suppressor. Nature Structural and Molecular Biology 2014, 21, 962-968, https://doi.org/10.1038/nsmb.2899.

13. Lomonosov, M.; Anand, S.; Sangrithi, M.; Davies, R.; Venkitaraman, A.R. Stabilization of stalled DNA replication forks by the BRCA2 breast cancer susceptibility protein. Genes and Development 2003, 17, 30173022, https://doi.org/10.1101/gad.279003.

14. Schlacher, K.; Christ, N.; Siaud, N.; Egashira, A.; Wu, H.; Jasin, M. Double-strand break repair-independent role for BRCA2 in blocking stalled replication fork degradation by MRE11. Cell 2011, 145, 529-542, https://doi.org/10.1016/j.cell.2011.03.041.

15. Roy, A.; Bhatia, K.S. In silico analysis of plumbagin against cyclin-dependent kinases receptor. Vegetos 2021, 34, 50-56, https://doi.org/10.1007/s42535-020-00169-8.

16. Garg, S.; Roy, A. In silico analysis of selected alkaloids against main protease (Mpro) of SARS-CoV-2. Chemico-Biological Interactions 2020, 332, 109309, https://doi.org/10.1016/j.cbi.2020.109309.

17. Garg, S.; Anand, A.; Lamba, Y.; Roy, A. Molecular docking analysis of selected phytochemicals against SARS-CoV-2 M pro receptor. Vegetos 2020, 33, 766-781, https://doi.org/10.1007/s42535-020-00162-1.

18. Ram, G.; Sharma, V.R.; Sheikh, I.; Sankhyan, A.; Aggarwal, D.; Sharma, A.K. Anti-cancer potential of natural products: recent trends, scope and relevance. Letters in Applied NanoBioScience 2020, 9, 902-907, https://doi.org/10.33263/LIANBS91.902907.

19. Agarwal, H.; Malviya, R.; Kumar Sharma, P. Evaluation of in situ gel forming system loaded with etoricoxib and herbal adjuvant nanoparticles against human colon cancer cell lines (HT-29). Letters in Applied NanoBioScience 2020, 9, 789-795, https://doi.org/10.33263/LIANBS0091.789795.

20. Roy, A. Hairy Root Culture an Alternative for Bioactive Compound Production from Medicinal Plants.

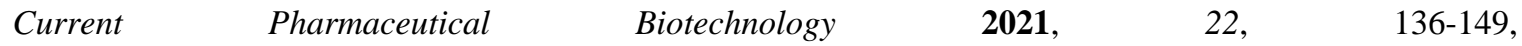
https://doi.org/10.2174/1389201021666201229110625.

21. Roy, A.; Bharadvaja, N. Venom-derived bioactive compounds as potential anti-cancer agents: a review. International Journal of Peptide Research and Therapeutics 2021, 27, 129-147, https://doi.org/10.1007/s10989-020-10073-z.

22. Sachdeva, V.; Roy, A.; Bharadvaja, N. Current prospects of nutraceuticals: A review. Current pharmaceutical biotechnology 2020, 21, 884-896, https://doi.org/10.2174/1389201021666200130113441.

23. Roy, A. Plumbagin: A potential anti-cancer compound. Mini reviews in medicinal chemistry, 2021, 21, 731737, https://doi.org/10.2174/1389557520666201116144421.

24. Roy, A.; Bharadvaja, N. Medicinal plants in the management of cancer: a review. International Journal of Complementary and Alternative Medicine 2017, 9, 00291, https://doi.org/10.15406/ijcam.2017.09.00291.

25. Khatoon, E.; Banik, K.; Harsha, C.; Sailo, B.L.; Thakur, K.K.; Khwairakpam, A.D.; Kunnumakkara, A.B. Phytochemicals in cancer cell chemosensitization: Current knowledge and future perspectives. In Seminars in Cancer Biology, 2020, S1044-579X(20)30150-4, https://doi.org/10.1016/j.semcancer.2020.06.014.

26. Qawoogha, S.S.; Shahiwala, A. Identification of potential anti-cancer phytochemicals against colorectal cancer by structure-based docking studies. Journal of Receptors and Signal Transduction 2020,40, 67-76, https://doi.org/10.1080/10799893.2020.1715431.

27. Prabhavathi, H.; Dasegowda, K.R.; Renukananda, K.H.; Lingaraju, K.; Naika, H.R. Exploration and evaluation of bioactive phytocompounds against BRCA proteins by in silico approach. Journal of Biomolecular Structure and Dynamics 2020, 1-15, https://doi.org/10.1080/07391102.2020.1790424.

28. Baseer, S.; Khan, S.; Nouroz, F. Computational drug Cumintrazole-analog for the treatment of mutant BRCA1 and BRCA2 proteins in breast cancer. International Current Pharmaceutical Journal 2015, 4, 410414, https://doi.org/10.3329/icpj.v4i8.24021. 